\title{
SI LE CONCEPT DE CRISE EST CONCEVABLE, ALORS...
}

\author{
Author(s) / Auteur(s) : \\ Jean-Louis LE MOIGNE \\ Professeur émérite \\ Aix-Marseille Université \\ Réseau Intelligence de la Complexité MCX-APC \\ jl.le-moigne@univ-amu.fr
}

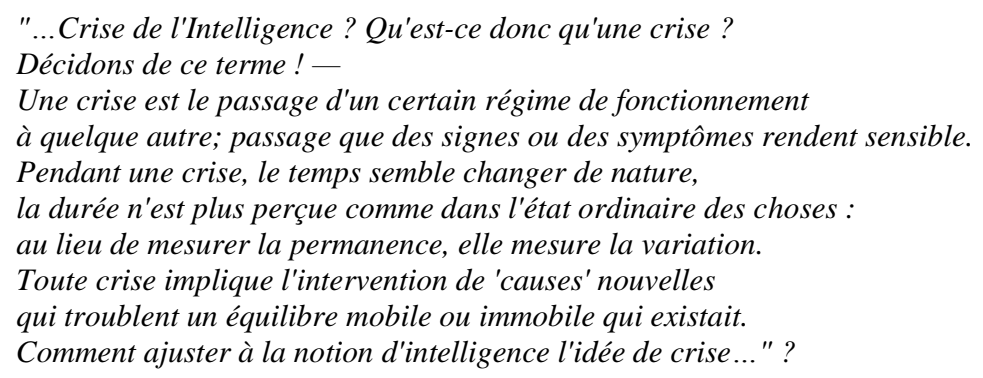

Paul Valéry, 1935.

Permettez moi de contribuer à cette réflexion collective sur une vision de 'La Crise' et des crises qui soit systemique et exploratoire et par là, moins analytique et déterministe voire prévisionniste - vision qui fut qui fut longtemps prégnante dans la culture des scientifiques et des praticiens - en l'ouvrant par la citation de quelques extraits de l'introduction de l'article séminal publié en 1975 par Edgar Morin sous le titre "Pour une Crisologie", article synthétisant un numéro de la revue Communications consacré à "La notion de crise".

"La notion de crise s'est répandue au $\mathrm{XX}^{\mathrm{e}}$ siècle à tous les horizons de la conscience contemporaine. Il n'est pas de domaine ou de problème qui ne soit hanté par l'idée de crise : le capitalisme, la société, le couple, la famille, les valeurs, la jeunesse, la science, le droit, la civilisation, l'humanité ... Mais cette notion, en se généralisant, s'est comme vidée de l'intérieur. [...] Aujourd'hui crise signifie indécision. C'est le moment où, en même temps qu'une perturbation, surgissent les incertitudes. [...] Mais dès qu'elle s'élargit à la culture, la civilisation, l'humanité, la notion perd tout contour. Elle permet tout au plus de dire que quelque chose ne va pas, mais l'information qu'elle donne se paie par l'obscurcissement généralisé de la notion de crise. Le mot sert désormais à nommer l'innommable ; il renvoie à une double béance : béance dans notre savoir (au cœur même du terme de crise) ; béance dans la réalité sociale elle-même où apparaît la 'crise'. [...]

Le problème clé est celui-ci : comment éclairer le concept de crise ? Comment le rendre éclairant ? (en sachant bien entendu que tout éclairage apporte sa propre ombre, que toute élucidation comporte sa propre tache aveugle). Tout d'abord, dans quel champ allons-nous considérer la notion de crise ? [...] En effet, si on veut, pour concevoir la crise, aller au-delà de l'idée de perturbation, d'épreuve, de rupture d'équilibre, il faut concevoir la société comme système capable d'avoir des crises, c'est-à-dire poser trois ordres de principes, le premier systémique, le second cybernétique, le troisième néguentropique; sans quoi la théorie de la société est insuffisante et la notion de crise inconcevable [...]." 
Éclairer d'emblée le concept de crise alors que nos dictionnaires ne parviennent à le définir qu'en énumérant les états ou les comportements perçus tenus pour non habituels de phénomènes perçus dans des centaines de contextes très différents, est ce une initiative plausible ? Le mot serait alors une sorte de préfixe pouvant être associé à chaque type de phénomène et exprimant qu'il est, ou qu'il fut ou qu'il a été dans une situation spécifique tenue pour inhabituelle et souvent tenue pour fâcheuse ; De la crise de fou rire à la crise de rage, de la crise de la famille, à celles du commerce international, celle de la crise du chômage ou celle du logement, les contextes sont si divers que le traitement de 'La Crise' semble devoir être spécifique, assuré par des experts spécialisés.

Experts pour lesquels la tentation de proposer l'application de leur prescription laquelle est argumentés par des diagnostics sommaires en arguant du fait que les acteurs concernés sont souvent censés réduire l'intelligible au simplifié. N'auront-ils pas à appliquer plutôt qu'à réfléchir, sans avoir à s'interroger sur le but qui a justifié le choix du ou des moyens prescrits, habituellement celui de la mise en place de nouveaux moyens censés eux 'résoudre' durablement la crise. Nouveaux moyens qui, légitimés par leur caractère endogène, spécifique de ce 'système en crise' risque très souvent de provoquer de nouvelles crises dans les systèmes avec lesquels il est ou peut-être en interaction ? N'est-il pas lui-même inséré dans un tapis de processus enchevêtrés?

C'est ce constat qui conduit à prendre conscience des inextricables interactions qui inter-relient "pour le meilleur et pour le pire" tous les phénomènes (ou processus) au sein desquels s'activent les sociétés humaines et plus généralement tous les systèmes actifs se différenciant en s'autonomisant tout en se solidarisant... 'Bon gré, mal gré'. C'est par leur activité identifiable ('Qu'est ce que cela fait ? Dans quoi ? Pour quoi ? Devenant qui ?') que nous pouvons les appréhender et les modéliser par la médiation artefactuelle de ce 'Pattern' qu'est le concept de Système General. Ce nœud d'interactions, en s'activant, va tendre à se stabiliser plus ou moins fugacement, tout en oscillant entre les deux extrêmes que sont la stabilité absolue et l'anarchie incompréhensible ; entre l'Ordre et le Désordre.

J'emprunte ici une image proposée par Paul Valéry en l'adaptant à la formation d'une organisation se stabilisant plus ou moins durablement: "Comme il faut une différence des températures des sources pour qu'une une machine, s'active, ainsi faut-il une différence d'ordre - désordre pour que s'active une organisation. Tout ordre ou tout désordre et rien ne va" (Valery, 1929, p.1015).

C'est sur une métaphore de ce type (emboitant les 'principes néguentropique, cybernétique et systemique') ce qu'Edgar Morin dégagera au fil des tomes successifs de la Méthode l' "incompressible paradigme de l'organisation" (Morin, 1980, p.351). Il ajoutera : "Un paradigme n'explique pas mais permet l'explication".

Cette conception de l'organisation d'un système s'ordonnant à la fois de façon autonomisante (à la fois organisée et s'organisant et par là en activité productrice, celle du processus, et en génération régénération), en interaction permanente avec ses environnements - ses éco-systèmes qu'il affecte et qui l'affectent dans ses comportements, suscitant à la fois les processus endogène d'émergence et de différentiation, ou de coagulations et uniformisations) - va permettre de rendre compte des tensions multiples qui imprègnent en permanence l'activité de toute organisation de systèmes entendus dans leur complexité ; Autrement dit, dans et par l'activité de tous types d'organisations sociales se formant sous la contrainte de nécessités vitales et par l'aspiration humaine à tenter d'explorer 'le champ des possibles', suscitant les 'projets' qui s'explicitent à fil de l'action des multiples 'faire en commun'.

Dès lors vont apparaître un peu plus intelligibles les multiples occurrences de 'crises' de tous types passibles d'investigations. Elles vont être suscitées par les effets des antagonismes endogènes et exogènes que suscite l'activité de toute organisation de systèmes s'ouvrant, systèmes vivant, système humains, systèmes sociaux, de tous types.

Antagonismes qu'accentuent la prise en compte des processus d'engrammation des 'traces' des myriades d'interactions qui se manifestent en particulier dans les organisations sociales ; Activant des processus endogènes de 'codage' par symbolisation-mémorisation organisationnelles, ils permettant le développement des processus de 'computation de symboles' et de communication nourrissant les processus d'interprétations par lesquels se forment les processus cognitifs d'exploration et de conception constitutifs de la formation des processus de decisions organisationnels; Lesquels pourront 
modifier la formation de crises selon que la rupture de stabilité organisationnelle diagnostiquée sera tenue pour une péripétie contingente ou une crise structurelle profonde ! (Edgar Morin développe peu cet argument dans son article (publié en 1975, année où Hervert A Simon publie sa conférence pour l'AMC Turing Award Lecture : "Computer Science as Empirical Inquiry: Symbol and Search"), mais il va être progressivement déployé dans les deux premiers tomes de la Méthode (1977-1980) dans lesquels s'argumente de façon constitutive la formation de "l'incompressible paradigme de l'organisation" (titre du premier chapitre de la derniere partie du tome 2).

L'exploration se forme souvent par la référence usuelle à la capacité de l'organisation à tenter de générer sa stabilisation de façon endogène en développant des dispositifs de régulation ramenant à une norme donnée telle et telle de ses activités ; Ces activités de feed back négatifs sont familières, mais elles peuvent inciter à développer des mécanismes antagonistes de feed back positifs par le développement en parallèle d'autres dispositifs de régulation concernant d'autres processus connexes. Déviances qui s'amplifient en se nourrissant de leur propre développement, entrainant la perte de stabilité que l'organisation voulait initialement assurer : Paradoxe classique de l'organisation, qu'Edgar Morin synthétise. "Pas d'organisation sans anti organisation, antagonisme et complémentarité sont les deux pole d'une même réalité complexe. La complexité est ce qui nous contraint à associer des notions qui apparemment devraient s'exclure. Toute organisation [...] porte en elle cette complexité puisque les relations entre les constituants, entre le tout et les parties, sont à la fois complémentaires, concurrentes et antagonistes". Raisonner en excluant le tiers est-ce alors bien raisonner dans les affaires humaines? (Simon, 1983)

Au balayage des facteurs organisationnels qui contribuent à rendre intelligible la formation des crises organisationnelles, Edgar Morin ajoute d'autres arguments dont celui de la multiplication des "doublebind", que l'on ne peut ici que mentionner, mais que l'on souligne car il met en valeur "le caractère multiple et multidimensionnel du dérèglement en chaîne, c'est-à-dire la variété des effets de crises mais aussi les formes crisiques de réponse..."

Sans pouvoir ici encore pousser plus avant l'effort de théorisation de cet étrange phénomène dont les bases sont déjà campées depuis plus de quarante ans dans l'appel d'Edgar Morin à une "Crisologie", nous pouvons collectivement nous exercer à l'approfondissement "de la crise de la conscience pour enfin faire émerger la conscience de la crise". N'est-il pas étrange que la crise devenant une réalité de plus en plus évidente, un terme de plus en plus multiplement employé, demeure un mot aussi creux ? [...] Ce terme diagnostic a perdu toute vertu explicative", alors "qu'il met en marche, ne serait-ce qu'un moment, tout ce qui peut apporter à notre intelligence des changements, transformations, évolution."

"Cette crise du concept de crise n'est-elle pas le début de la théorie de la crise ?" espérait alors Edgar Morin en achevant son article. Convenons, hélas, qu'à ce jour notre compréhension de la formation et de l'évolution des phénomènes crisique n'a guère progressé. Nos cultures ne sont guère accoutumées encore aux raisonnements dialogiques ni aux explorations intentionnelles des contextes ouverts (les 'tapis de processus') dans lesquels s'élaborent des stratégies des organisations sociales. La logique du plausible. (J-C Gardin), et Les mathématiques du raisonnement plausible (G Polya) ou la Science de Conception (The science of Design) ou La théorie de l'enquête (J Dewey) comme toutes les démarches d'ouverture critique de la rationalité projective, celle du raisonnement à fin de désacralisant sans l'exclure le raisonnement à cause de... démarches délibératives 'procedural rationality') qui caractérisent le champ de la pensée complexe, ne nous sont pas encore assez familières.

Si bien que dans le jeu - et souvent dans le feu - de l'élaboration des decisions dites stratégiques, nous préférons nous attacher à la prévision ou à l'anticipation qu'à la compréhension de ces phénomènes à la fois étranges et familiers que sont les crises ; Crises dont nous voudrions prévoir ou prédire les éventuelles conséquences, les potentialités en termes de risques (une risquologie selon Bouilloud, 2012) plutôt que de privilégier leur compréhension, leur formations et leur effectivité.

Si les praticiens réfléchissant (Schön, 1983) s'attachent à "faire pour comprendre ET à comprendre pour faire"1, alors...

${ }^{1}$ P Valéry attribue cette devise à Léonard de Vinci en lisant une traduction de ses Carnets (in 'Vues', 1948) 


\section{RÉFÉRENCES}

BOUILLOUD, Jean-Philippe (2012). "De la 'crisologie' à la 'risquologie'". Communications, 2012/2, n91, pp.153-159.

VALÉRY, Paul (1935). Propos sur l'intelligence.

VALÉRY, Paul (1973). Les cahiers I (1894-1945). Paris : Gallimard (coll. Pléiade), éd.2010.

MORIN, Edgar (1980). La méthode 2 : La Vie de la Vie. Paris : Seuil (coll. Points).

SIMON, Herbert A. (1983). Reason in Human Affairs. Stanford University Press.

SCHÖN, Donald A. (1983). Le praticien réflexif : À la recherche du savoir caché dans l'agir professionnel. Trad. Heynemand J. \& Gagnon D. [The Reflective Practitioner: How Professionals think in Action. New York: Harper Collins]. Montréal : Les Éditions Logiques (coll. Formation des maîtres), éd.1994. 